\title{
An Interdisciplinary Approach to the Management of Basal Cell Carcinoma of the Head and Neck
}

\author{
SHAN R. BAKER, M.D., F.A.C.S. \\ NEIL A. SWANSON, M.D. \\ ROY C. GREKIN, M.D.
}

ARTICLE

\begin{abstract}
At the University of Michigan the dermatologic surgeon works closely with the head and neck surgeon in resecting extensive cutaneous malignancies that could benefit from the combined skills of both surgical specialists. Mohs surgery offers complete microscopic controlled resection of the cutaneous portion of skin cancers. Tumors extending deeply from the skin into underlying bone and soft tissue are resected with the assistance of the head and neck surgeon familiar with the anatomy and trained in the protection of the vital structures of the head and neck. It is evident that patients with large or aggressive basal cell carcinomas will best be served when this interdisciplinary approach has become commonplace.
\end{abstract}

The past decade has increasingly demonstrated the advantage of an interdisciplinary team approach to the management of certain diseases of the head and neck. The head and neck surgeon has combined his skills with those of his neurosurgical colleagues to

Shan R. Baker, M.D., F.A.C.S., Professor, Department of Otolaryngology-Head \& Neck Surgery; Neil A. Swanson, M.D., Associate Professor, Departments of Dermatology and Otolaryngology-Head \& Neck Surgery; and Roy C. Grekin, M.D., Assistant Professor, Department of Dermatology, are from the University of Michigan Hospitals, Ann Arbor, Michigan.

Address reprint requests to Shan R. Baker, M.D., F.A.C.S., Department of Otolaryngology, University of Michigan Hospitals, 1500 East Medical Center Drive, Ann Arbor, MI 48109.

Presented at the annual meeting of the American Academy of Facial Plastic and Reconstructive Surgery, September 12, 1986, San Antonio, Texas.

J. Dermatol. Surg. Oncol. 13:10 October 1987 perform craniofacial resections of tumors extending to regions of the anterior skull base. Likewise, neurotologists have teamed up with neurosurgeons to resect extensive posterior and middle cranial fossa tumors, which overall has resulted in improved function postoperatively. It is only logical that the Mohs surgeon seek the skills of the head and neck surgeon to assist him in resecting extensive facial skin cancers that extend into the nasal passage, paranasal sinuses, or orbital region. ${ }^{1}$ Such extension may require the assistance of a head and neck surgeon to adequately remove the neoplasm extending into the deeper planes of the face and neck. Similarly, extensive facial skin cancers may invade deeply to involve the cranium and occasionally the underlying dura mater and brain. Such cases call for the assistance of surgeons trained in neurosurgery.

At the University of Michigan the dermatologic surgeon works closely with the head and neck surgeon in resecting extensive cutaneous malignancies that could benefit from combining the skills of both surgical specialists. ${ }^{2}$ Tumors requiring this multidisciplinary approach are those demonstrating aggressive biologic behavior. Such tumors frequently occur in the " $\mathrm{H}$ " zone of the face (Fig. 1). This zone is noted for its high incidence of recalcitrant basal cell carcinomas (BCCs), and includes the central midfacial area including the upper lip, nose, and medial canthal regions. The zone extends horizontally to incorporate the lower eyelids extending to the preauricular area. The vertical portion of the " $\mathrm{H}$ " shaped zone of the facial skin is a large area of skin in the preauricular area extending superiorly into the temple and inferiorly toward the angle of the mandible. This area also includes portions of

1095 

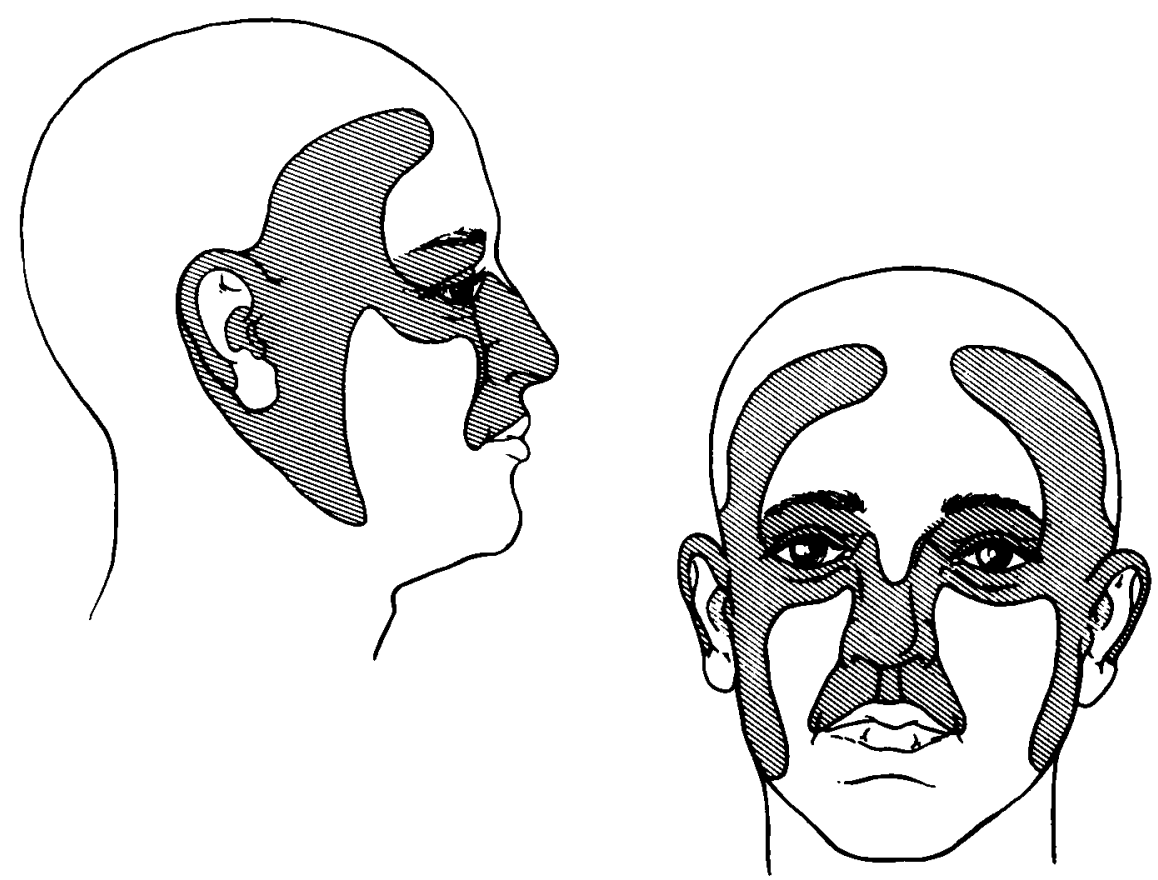

FIGURE 1. The " $\mathrm{H}$ "shaped zone of the face is noted for its high incidence of recalcitrant BCCs.

the auricle and postauricular skin. The " $\mathrm{H}$ " zone represents a region where $\mathrm{BCC}$ tends to be more invasive and destructive and to recur more frequently. ${ }^{3}$ Thus, it is more difficult to treat skin cancer in this region compared with similar tumors located in other sites about the face and neck.

The apparent major reason for the aggressiveness of skin cancers in the " $\mathrm{H}$ " zone is related to the fact that this region represents an area rich in embryologic fusion planes. Fusion planes are frequently perpendicular to the surface of facial skin and provide tissue interfaces for extension of tumor deeply toward underlying structures of the face. ${ }^{4}$ When this occurs, it is best for the dermatologic surgeon to seek the assistance of a head and neck surgeon to facilitate resecting tumor involving bone, muscle, and vital structures.

During the last 5 years, we have had extensive experience treating large facial skin malignancies by the interdisciplinary approach combining the skills of the dermatologic surgeon and the head and neck surgeon. The interdisciplinary approach allows for complete microscopic controlled excision of the tumor while maximizing conservation of tissue, and thus minimizing functional and cosmetic disability. The indications and techniques for the use of Mohs surgery in the treatment of skin cancer have been described. ${ }^{3}$ The purpose of this paper is to present representative cases that exemplify the usefulness 1096 of combining the surgical skills of these two surgical specialists.

\section{AURICULAR REGION}

In contrast to morpheaform BCCs that extend widely in a peripheral fashion, ulcerative $\mathrm{BCCs}$ tend to grow more deeply into the underlying structures of the face. BCCs occurring in the preauricular area are frequently of the ulcerative type and have a relatively high recurrence rate due to the tendency of the tumor to extend medially along the perichondrium of the external auditory canal. ${ }^{5}$ Robins ${ }^{6}$ reports the preauricular area as having statistically significantly higher recurrence rates following controlled microscopic excision of BCC compared with other anatomic regions of the face. The aggressive nature of $\mathrm{BCC}$ involving the preauricular area is represented by the patient in Figure 2. This patient presented with a neglected ulcerative $B C C$ of the preauricular skin. Figure 2 also demonstrates the skin defect following adequate removal of the tumor from the skin of the preauricular region. However, tumor extended medially in the subperichondrial plane of the cartilaginous external auditory canal toward the bony canal. Tumor extended beyond the confines of the canal anteriorly and necessitated a parotidectomy with 


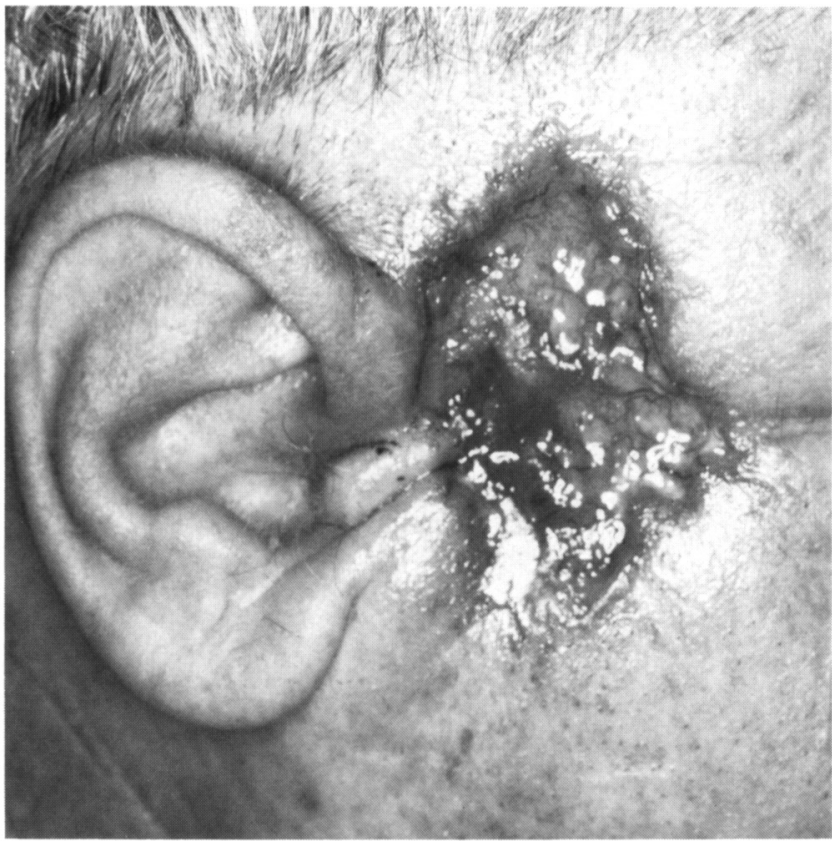

FIGURE 2A. Neglected ulcerative BCC of the preauricular skin.

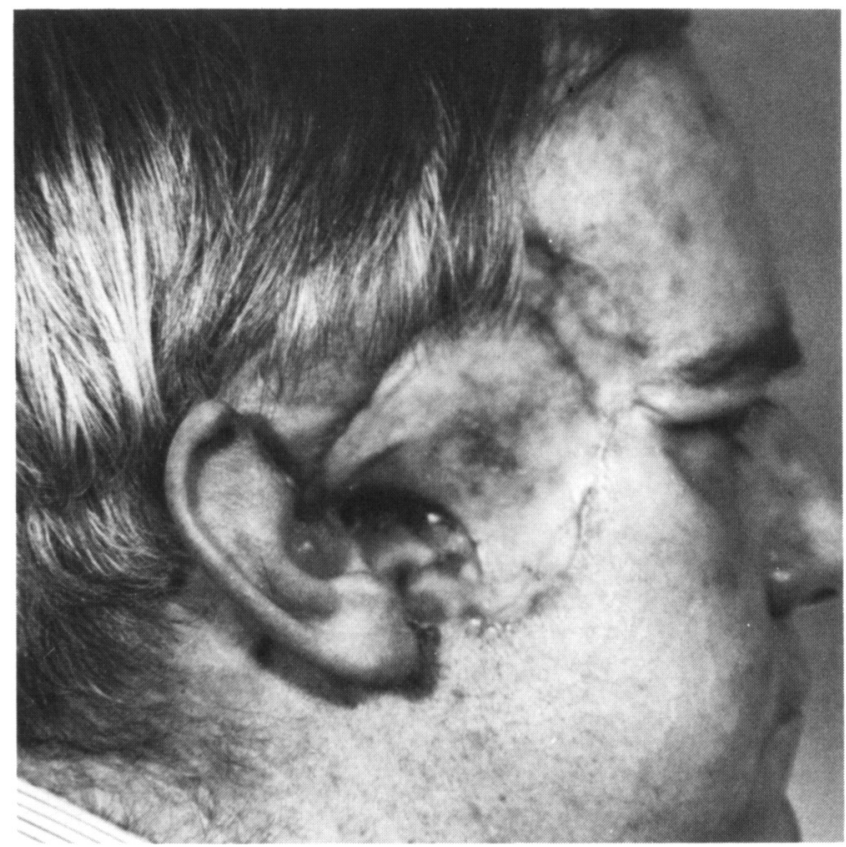

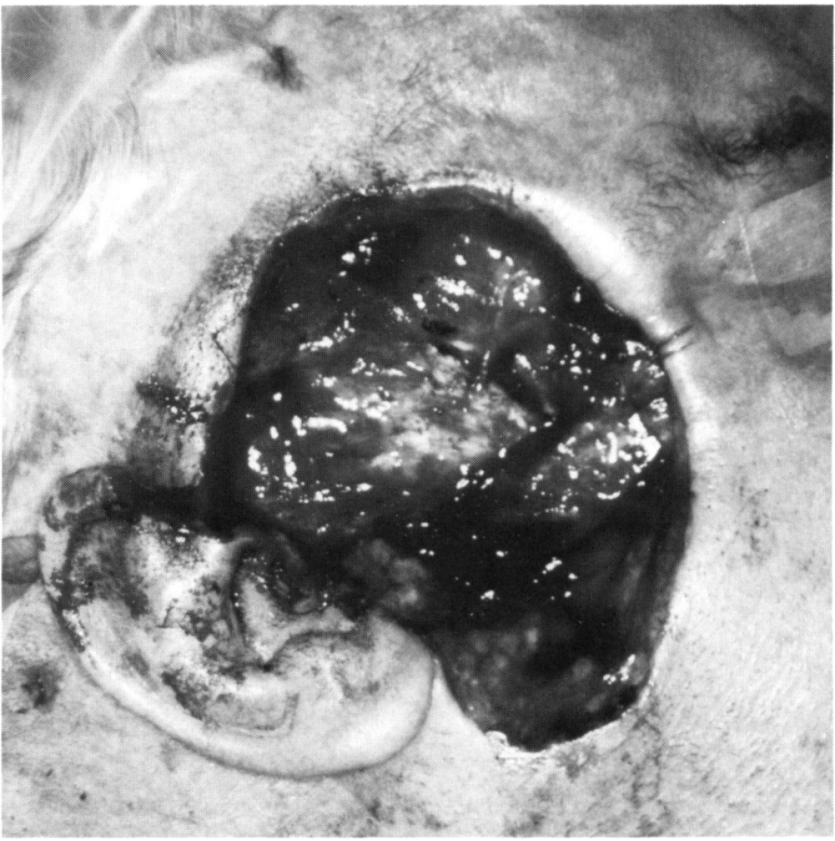

FIGURE 2B. Skin defect following Mohs excision of tumor.

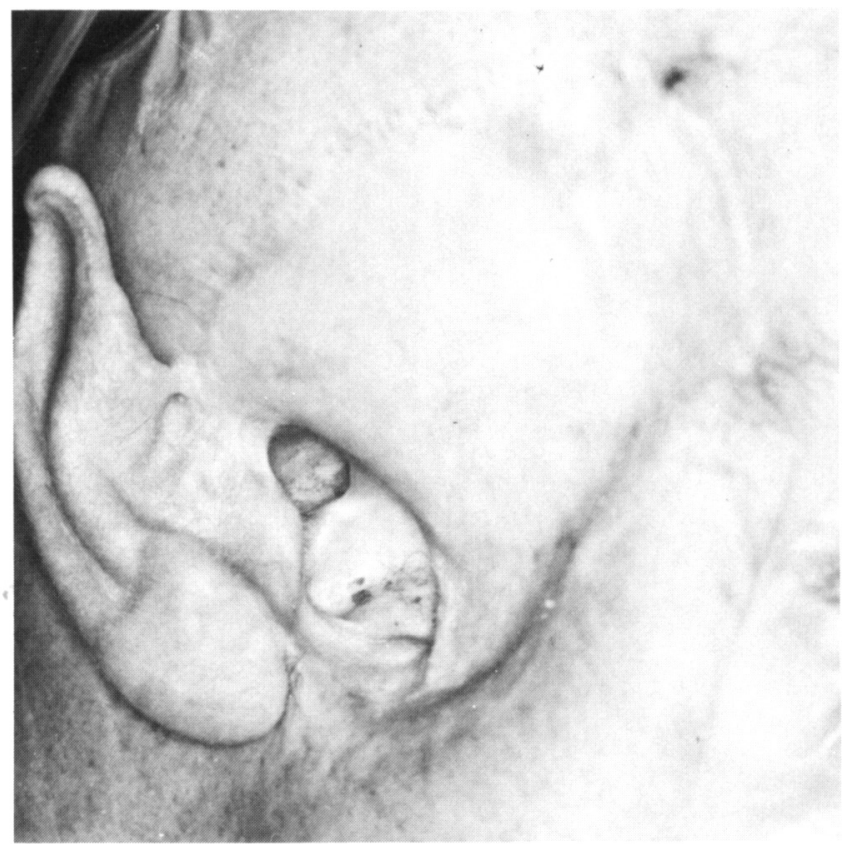

FIGURE 2C AND D. Extension of tumor medially required removal of the entire condyle of the mandible and the tympanic bone except bony anulus. Defect was covered with a split-thickness skin graft. One year postoperative.

preservation of the facial nerve. Extension of tumor medially toward the tympanic membrane required removal of the entire condyle of the mandible and tympanic bone down to, but not including, the bony anulus.

Extensive BCCs extending into the auditory canal sometimes require radical mastoidectomy

J. Dermatol. Surg. Oncol. 13:10 October 1987 with removal of the cartilaginous and bony external auditory canal. In such instances, the surgical approach is accomplished by making a standard tympanomeatal flap and elevating the skin of the external canal away from the anulus. Following skin elevation, the most medial aspect of the flap is sampled by the dermatologic surgeon to evaluate the

1097 

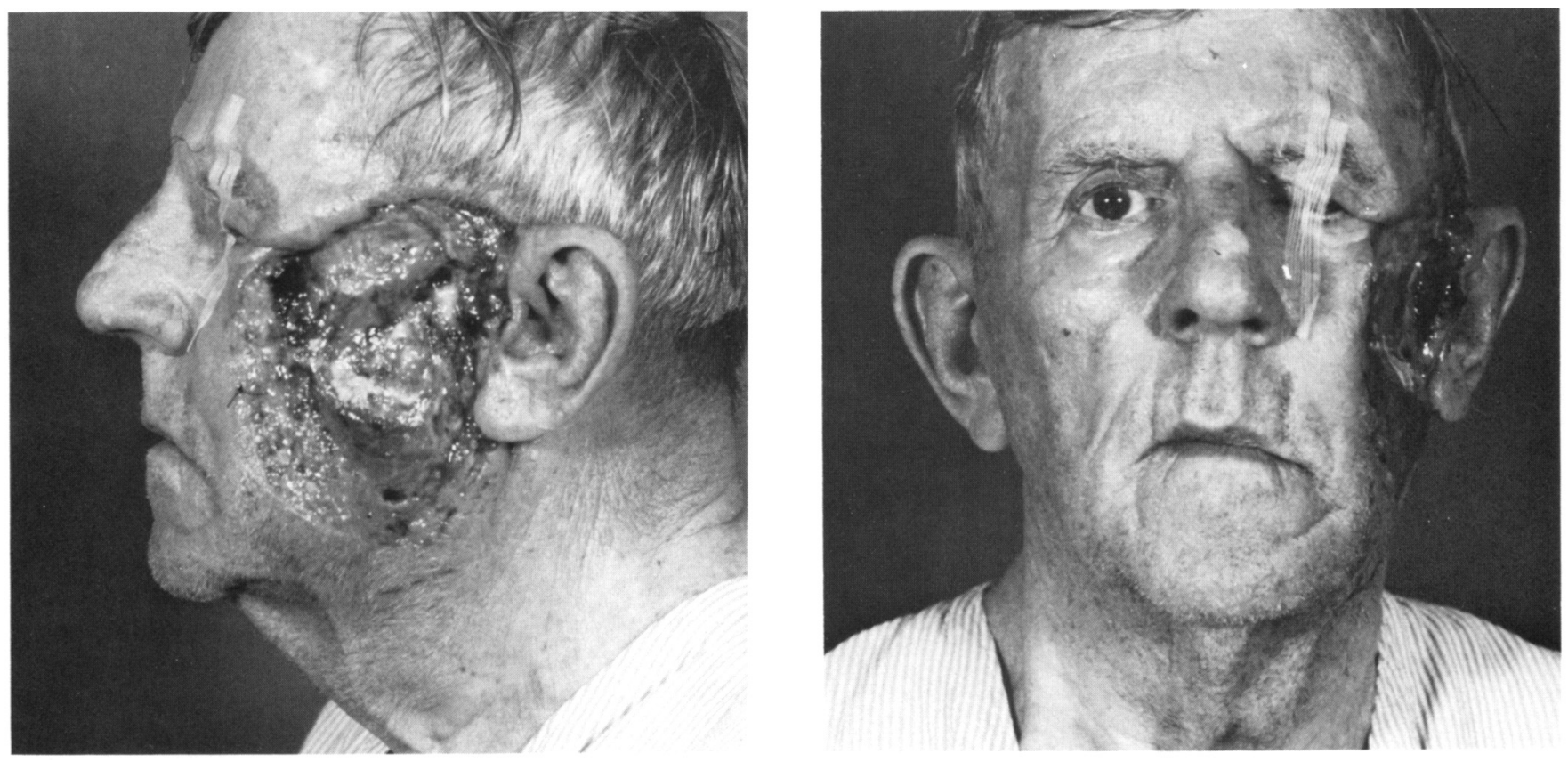

FIGURE 3A AND B. Following Mohs excision of BCC of the preauricular area necessitating removal of the left parotid gland and sacrifice of the facial nerve.

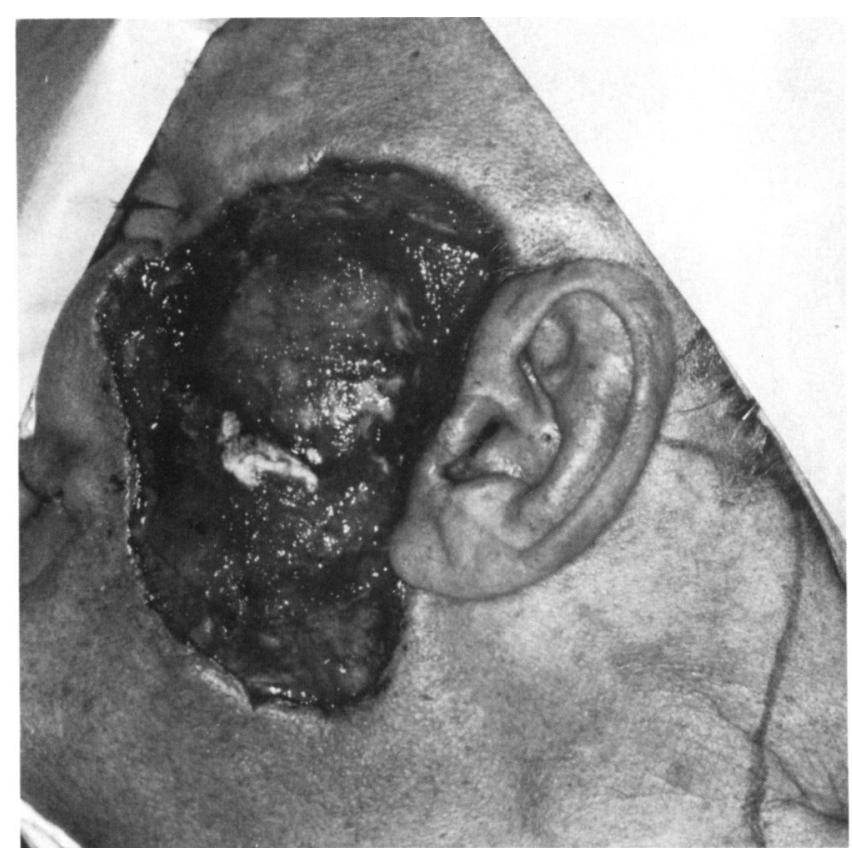

FIGURE 3C. Defect reconstructed with postauricular skin flap.

medial surgical margin. Should tumor be noted at the extreme medial aspect of the flap, it is recommended that the patient undergo removal of the tympanic membrane, annular ligament, and radical mastoidectomy. At the time of tympanic membrane removal, the mucous membrane in the middle ear medial and adjacent to the anulus is sampled and 1098

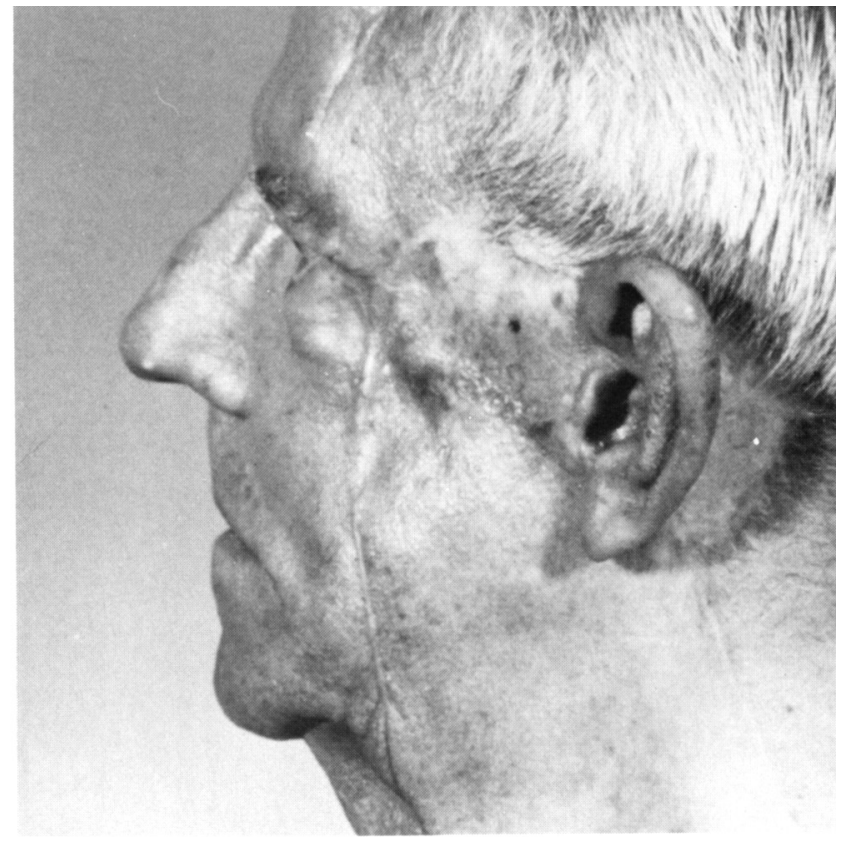

FIGURE 3D. Nine months postoperative.

examined for foci BCC. If the presence of tumor is documented in the middle ear, consideration must be given to a formal temporal bone resection or the utilization of postoperative radiotherapy.

Patients with BCC extending deeply from the preauricular area requiring removal of the external ear canal should probably be observed from 12 to

I. Dermatol. Surg. Oncol. 13:10 October 1987 
18 months by surfacing the area with a split-thickness skin graft rather than subjecting them to immediate reconstruction using a local or regional flap. This is particularly true if there is concern for the adequacy of surgical margins around the neoplasm. The thin covering offered by the skin graft may permit early detection of recurrent neoplasm (Fig. 2).

Figure 3 represents a patient that developed BCC of the preauricular area necessitating removal of the left parotid gland and sacrifice of the facial nerve. Tumor extended onto the zygomatic arch, and sampling of the periosteum by the dermatologic surgeon demonstrated tumor involvement. Tumor was noted to extend to the deep aspect of the zygomatic arch. Working with the assistance of the dermatologic surgeon who provided histologic assessment of the surgical margins, the head and neck surgeon removed the entire arch as well as the upper portion of the masseter muscle. It was the consensus of the surgeons involved that surgical margins were adequate to allow immediate reconstruction of the defect. The defect was reconstructed utilizing a cervical flap based inferiorly and harvested from the postauricular area.

A postauricular cervical cutaneous flap is advantageous for reconstruction of defects in the preauricular area because it provides the best color and texture match with that of the skin of the remaining cheek and forehead regions (Fig. 3). Another advantage is that the secondary defect, although often requiring a split-thickness skin graft to facilitate closure, is located in the postauricular area which is helpful for scar camouflage and overall cosmesis.

Whenever it is necessary to sacrifice the facial nerve in order to completely remove a $B C C$, attempts should be made to graft the portion of resected nerve. However, frequently tumors located in the preauricular area extend peripherally to such a degree that the distal branches of the facial nerve have to be sacrificed in order to ablate all of the tumor. Such instances preclude any attempt to reanimate the face by cable nerve grafting. Unfortunately, in most of these cases, there is a frequent need to remove the masseter muscle and portions of the temporalis muscle, thus precluding the possibility of rehabilitating the face with a dynamic sling. The only alternative in such cases may be the use of static fascia lata slings to suspend the oral commissure in a more symmetrical position.

Similar to BCCs arising in the preauricular skin, tumors occurring in the postauricular sulcus often grow in an aggressive manner extending through the conchal cartilage as well as spreading into the external auditory canal. However, tumor spread tends to be more in a superior and inferior direction. ${ }^{5}$ Tumor may spread inferiorly to involve the sternocleidomastoid muscle and deeper structures of the upper neck or spread medially to involve the cortex of the mastoid bone. Ceilley et al. ${ }^{7}$ reported a higher than usual recurrence rate in treating auricular BCCs and noted the frequent aggressiveness of tumors occurring in the postauricular region.

Ulcerative tumors located in the inferior aspect of the postauricular sulcus often extend inferiorly to involve the sternocleidomastoid muscle, tail of the parotid gland, and deeper structures of the neck. ${ }^{5}$ A representative case is shown in Figure 4. The patient presented with a recurrent ulcerative BCC of the lower postauricular sulcus that extended into the deep structures of the upper neck, necessitating a regional neck dissection confined to the upper posterior cervical triangle in order to irradicate the entire tumor. In patients requiring parotidectomy or regional neck dissection, the head and neck surgeon performing the operation has relied on the dermatologic surgeon to assist him in surveying all of the deep soft tissue margins, thus ensuring that all tumor has been eradicated from the deeper tissue planes of the neck. In this instance, the tail of the parotid gland, posterior digastric muscle, and upper sternocleidomastoid muscle were all histologically examined to ensure adequacy of tumor ablation in tissue planes deep to the skin (Fig. 4).

Along with location of BCCs, tumor histology plays an important role in the treatment of these cutaneous neoplasms. The more common tumor histologies (noduloulcerative, superficial) represent little problem in the diagnosis and treatment, particularly if the tumor is small and occurs outside of the " $\mathrm{H}$ " zone of the face. Those tumors with aggressive histologic behavior, however, often warrant a more exacting treatment of microscopic controlled resection. These histologic subgroups include the morpheaform or sclerotic BCC, multicentric infiltrating tumor, and the aggressive keratinizing BCC (basosquamous carcinoma or metatypical carcinoma).

A higher than usual recurrence rate for morpheaform $B C C$, even after treatment by Mohs technique, has been reported. This may result from subtle subclinical extension and possibly from stromal dependence of this tumor. Salasche and Amonette ${ }^{8}$ found subclinical extensions to average $7.2 \mathrm{~mm}$ in 51 morpheaform BCCs. They noted that the greatest degree of subclinical extension occurred in the preauricular region. Ceilley et al. ${ }^{7}$ found that of their inadequately excised BCCs of the ear, the morpheaform type was the most common. 


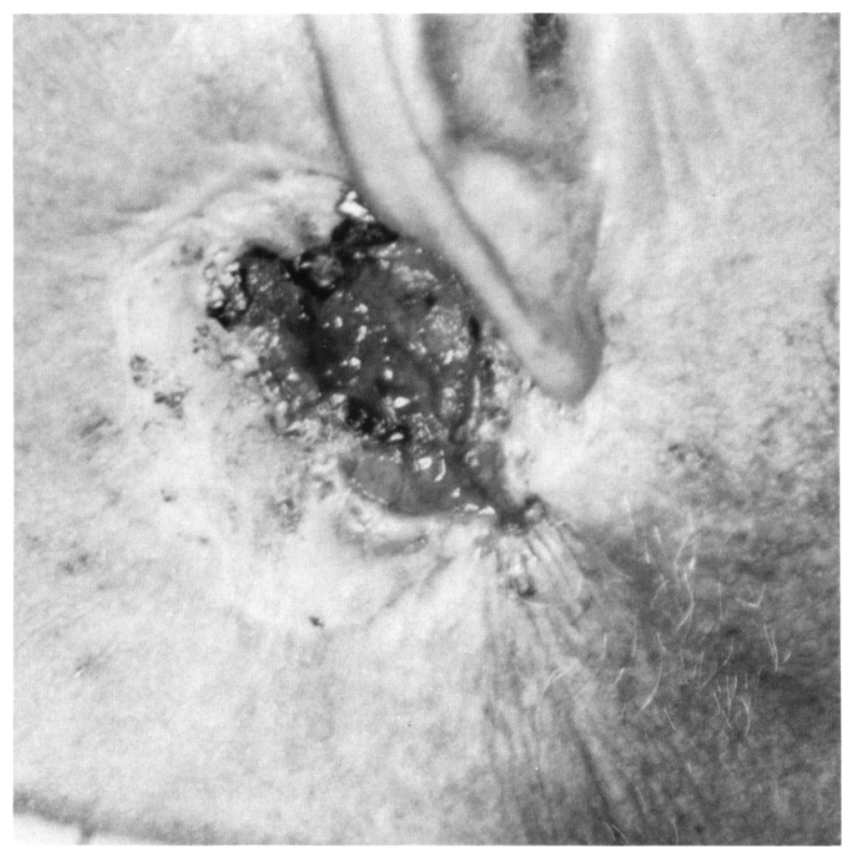

FIGURE 4A. Recurrent ulcerative BCC postauricular sulcus.

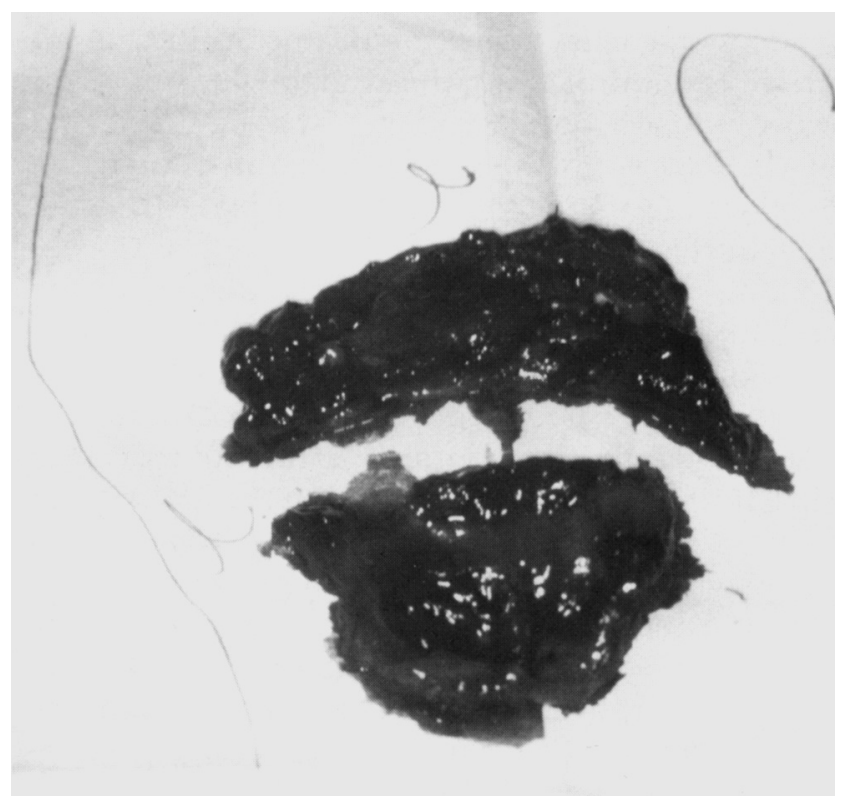

FIGURE 4C. Mohs surgical margin specimen following regional neck dissection, necessary to extirpate tumor extending deeply in the neck. This specimen represents the interface between the resected tumor and remaining normal tissues in the neck. Specimen was used for complete histologic control of surgical margin.

Figure 5 demonstrates a morpheaform $\mathrm{BCC}$ involving the preauricular skin. Surgical excision utilizing microscopic controlled resection necessitated removal of the posterior facial skin, most of the skin of the upper neck and postauricular area, as well 1100

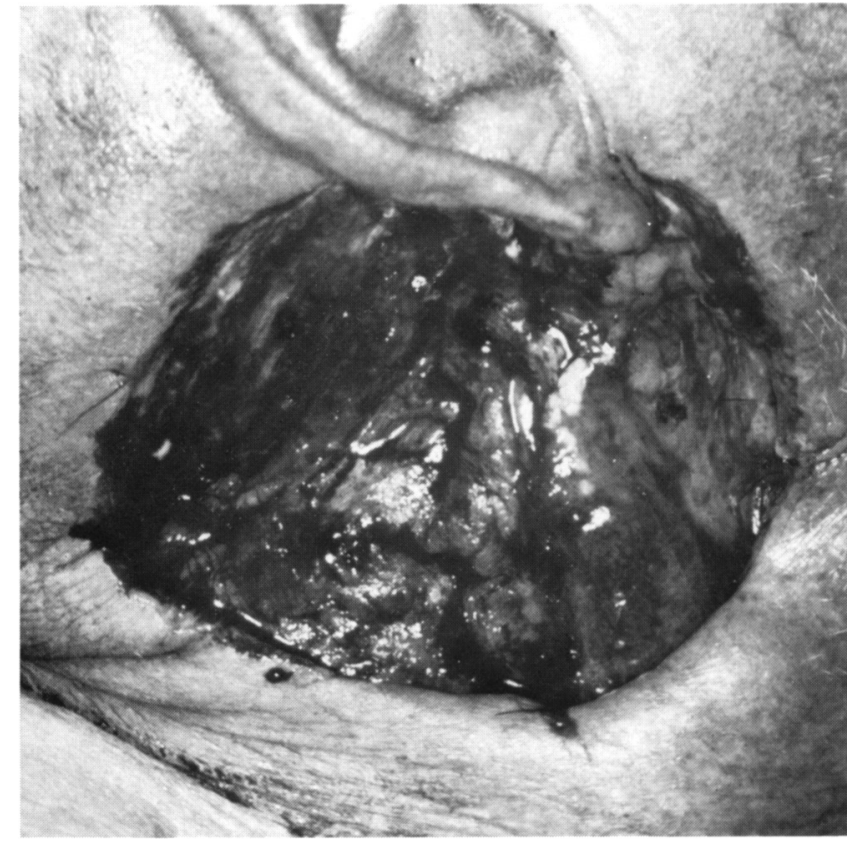

FIGURE 4B. Following Mohs excision of cutaneous portion of tumor.

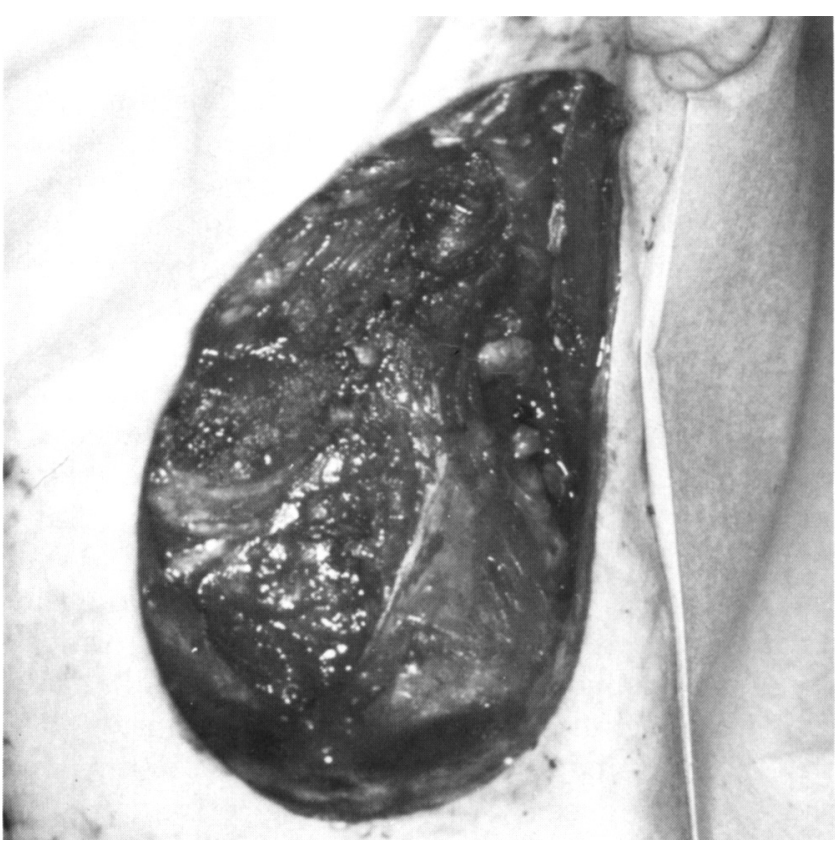

FIGURE 4D. Defect following upper posterior neck dissection.

as a large portion of the scalp located in the occipital area. This case nicely demonstrates the massive subclinical extension that can occur with morpheaform BCCs. It also demonstrates the usefulness of an interdisciplinary approach for the management

I. Dermatol. Surg. Oncol. 13:10 October 1987 


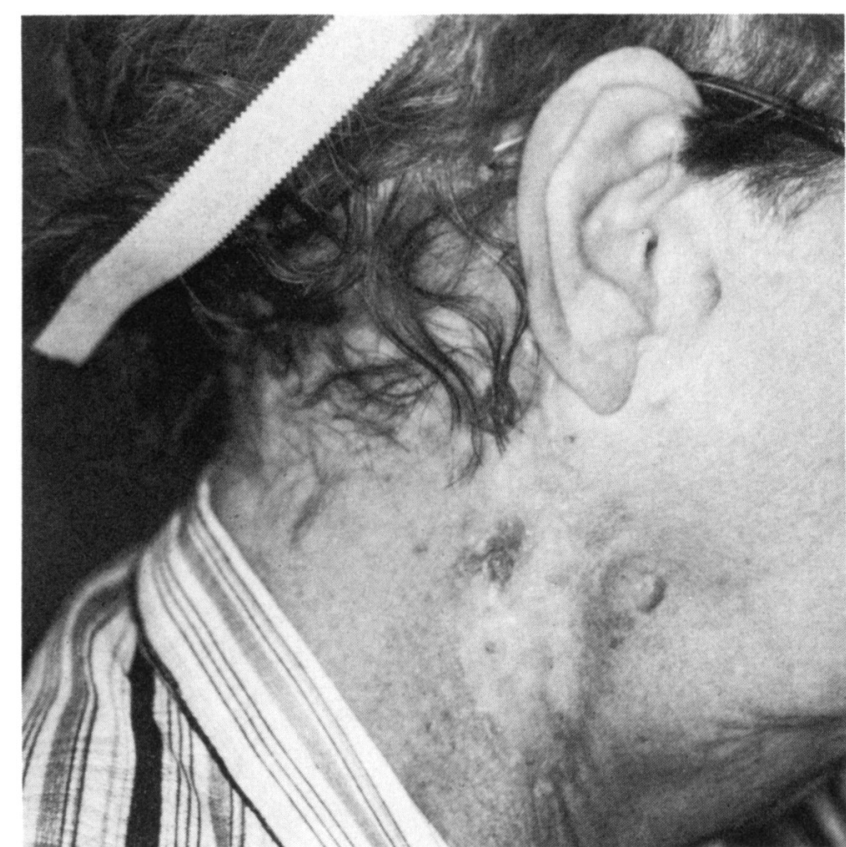

FIGURE 5A. Morpheaform BCC of periauricular skin.

of extensive cutaneous malignancies. The dermatologic surgeon can ensure adequacy of resection margins and the head and neck surgeon can provide technical assistance in resection of deep tissues and reconstruction of the ablative defect following ablative surgery.

Figure 6 demonstrates another patient suffering from a histologically aggressive tumor. The neoplasm presented as a keratotic (basosquamous) BCC located in the postauricular area with extension into the parotid gland. The patient required a radical parotidectomy and sacrifice of the facial nerve and facial nerve cable grafting. Because of the aggressiveness of the tumor, it was elected to administer postoperative radiotherapy, although all surgical margins were free of residual tumor.

The decision to combine postoperative radiotherapy with microscopically controlled surgical excision of extensive skin cancers is a matter of clinical judgment based on the size and location of tumor and the histology of the cancer. We have treated a small select group of patients suffering from BCC with postoperative radiation therapy in specific instances where recurrent tumor required extensive resection of the facial soft tissues and where surgical margins of normal tissue were not as abundant as we would desire. More specifically, postoperative radiotherapy has been used to treat potentially persistent microscopic disease in cases where cancer encroached on regions of the skull

J. Dermatol. Surg. Oncol. 13:10 October 1987

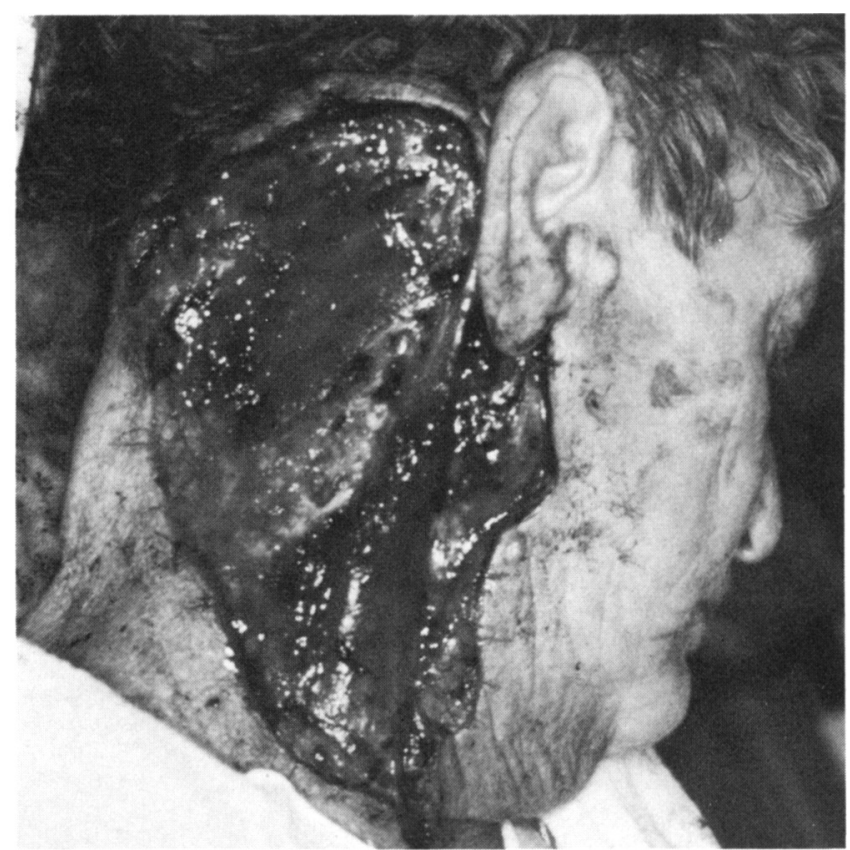

FIGURE 5B. Defect following Mohs excision.

base such as the glenoid fossa, jugular foramen, cribiform plate, and the middle skull base.

\section{CENTRAL FACE}

Mora and Robins ${ }^{9}$ showed that BCC in the central face, similar to those located in the para-auricular areas, tend to be more invasive, more destructive, and more recurrent than similar tumors located at other sites of the head and neck. The junction of the nasal ala with the upper lip and facial skin of the cheek represents an important embryologic fusion plane where BCCs may extend deeply into the underlying structures of the face including the nasal passage and paranasal sinuses. Tumor may also demonstrate subclinical extension along the perichondrium of the upper and lower lateral nasal cartilages into the depths of the nasal vestibule necessitating full-thickness resection of portions or of all the nasal tip and ala. ${ }^{10}$

A limited maxillectomy is occasionally required when midfacial skin cancer involves the pyriform aperture of the nose. This occurs when neglected or recurrent $B C C$ s occurring in the nasolabial crease and upper lip spread laterally along the periosteum covering the face of the lower maxilla. Neoplastic cells may also spread inward along the lateral wall of the nose, often beneath the inferior turbinate. When this occurs, the preferred treatment is com- 


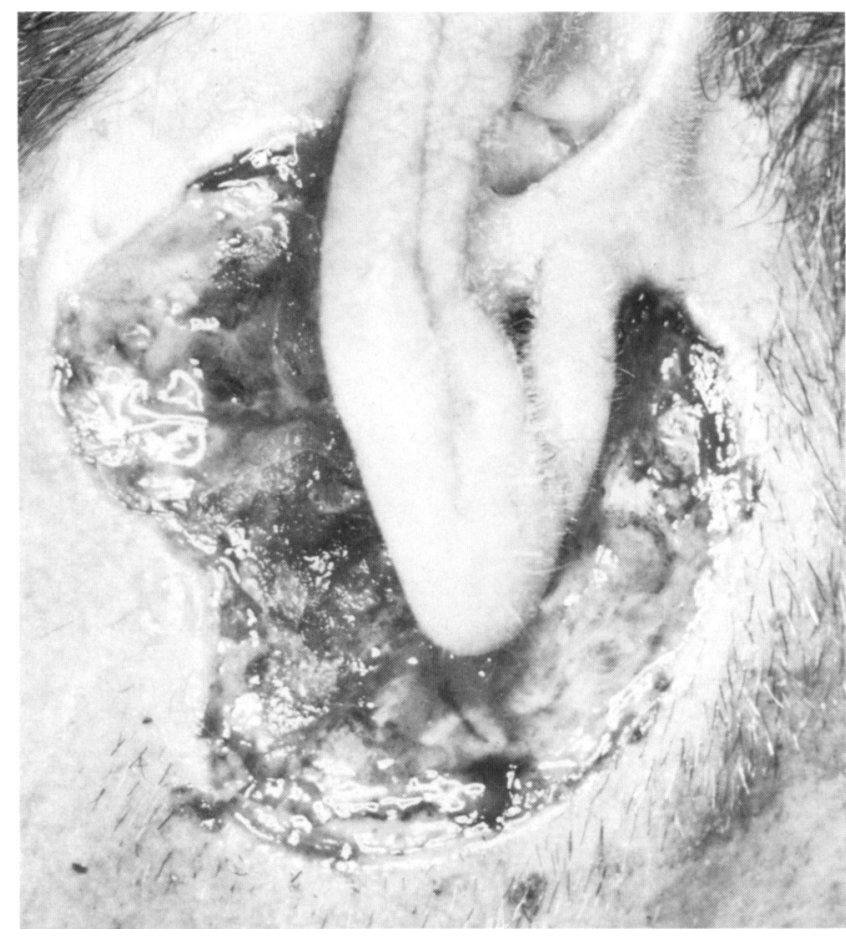

FIGURE 6A. Cutaneous malignancy of periauricular area.

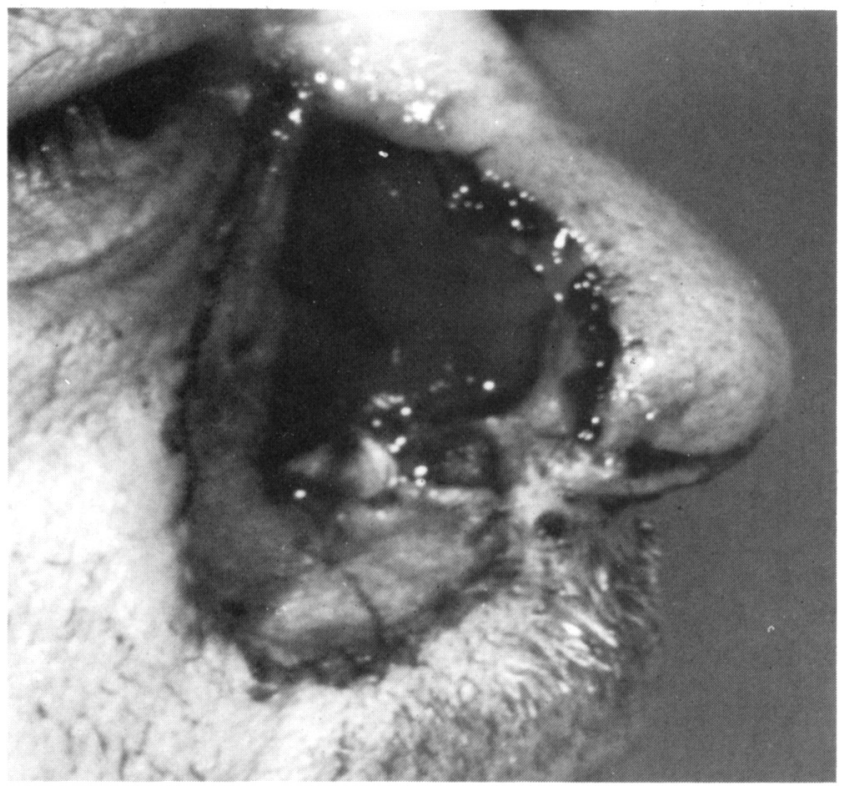

FIGURE 7. Cutaneous malignancy extending deeply into the lateral nasal passage may require complete removal of the bony structures in the vicinity of the nasal apperture.

plete removal of the bony structures in the vicinity of the nasal aperture. The anterior lateral wall of the nose and the medial aspect of the face of the maxilla are completely removed along with the in-

1102

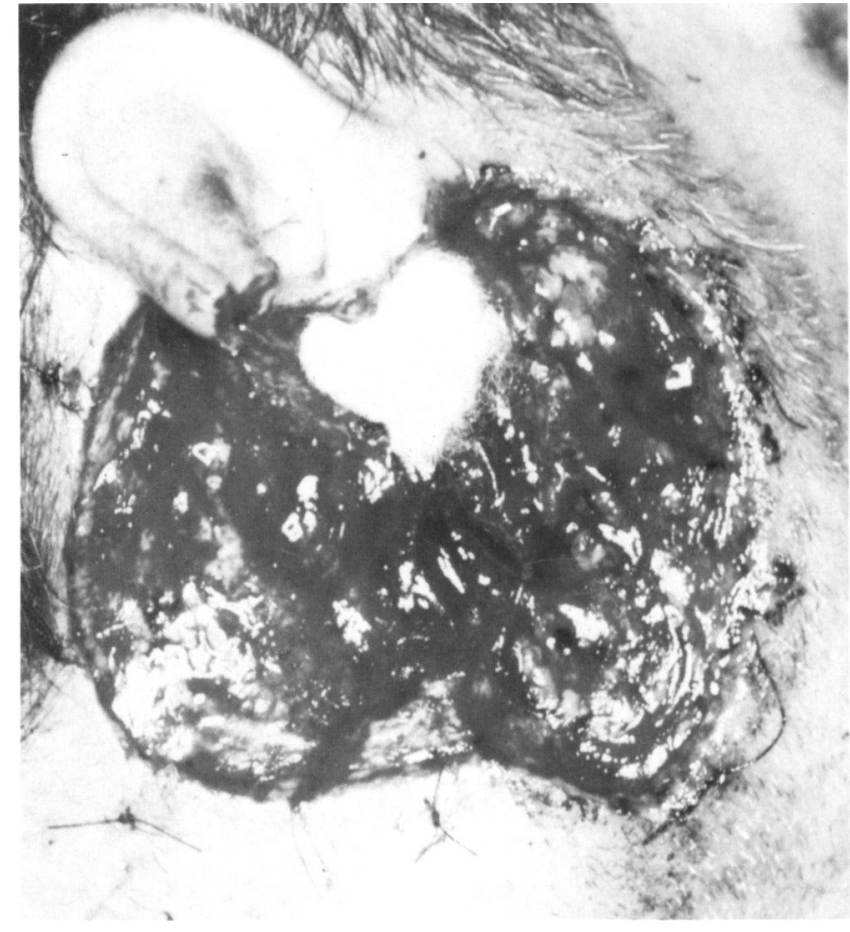

FIGURE 6B. Complete resection of tumor required radical parotidectomy, sacrifice of the facial nerve and facial nerve cable graft.

ferior aspect of the lacrimal duct and the anterior one-half of the inferior turbinate (Fig. 7). In addition to removal of the lateral aspect of the nose, a portion or all of the premaxilla may require removal. Such resections are facilitated by the cooperative efforts of the dermatologic surgeon and the head and neck surgeon.

Nasal passage and paranasal sinus involvement with facial skin cancer occurs not only as a result of neglected or recurrent $B C C$, but when tumors are biologically aggressive and are located in the midfacial region. Reconstruction after controlled microscopic surgical ablation of such neoplasms should be delayed 12 to 18 months in most instances to provide an opportunity for any persistent carcinoma to manifest itself. In the interim, the surgical defect can be covered with a split-thickness skin graft. A prosthesis can be manufactured to assist in the cosmetic and functional rehabilitation of the patient, and can be used until reconstruction is deemed advisable.

BCC occurring in the superior portions of the midface occur most frequently in the region of the medial canthus. Tumors located near the medial canthus of the eye may extend deeply into the orbit or the anterior ethmoid sinuses, necessitating resection of part or of all of the orbital contents and ethmoidectomy.

J. Dermatol. Surg. Oncol. 13:10 October 1987 


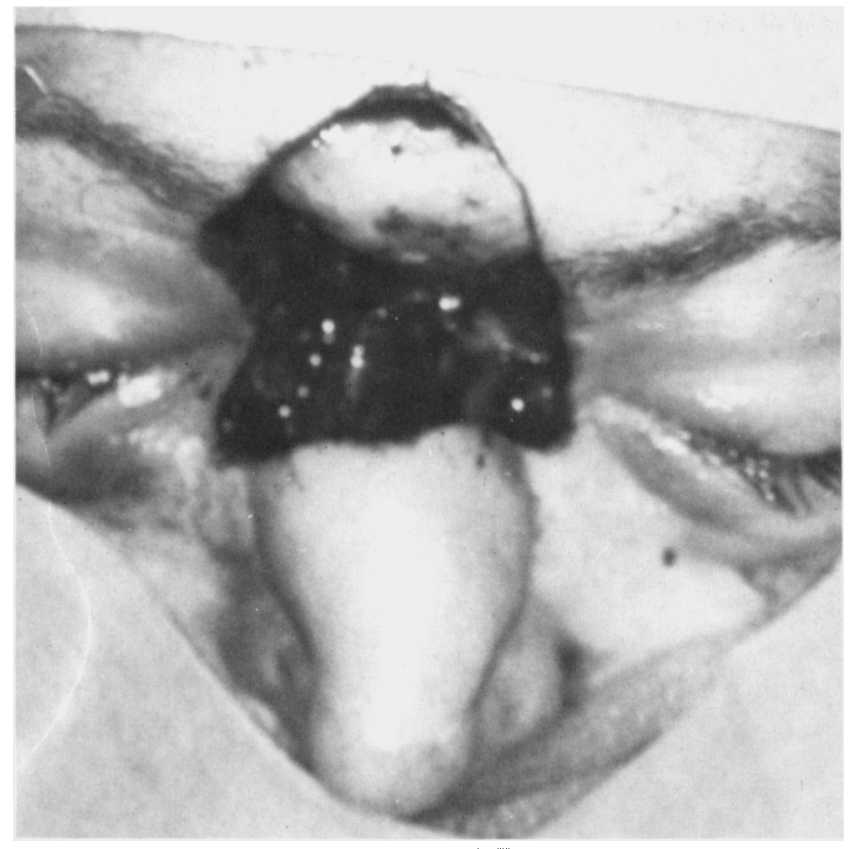

FIGURE 8A. Following Mohs excision of recurrent basal cell carcinoma.

Figure 8 represents a patient following Mohs excision of a recurrent $B C C$ located in the region of the medial canthal and glabella regions. At the time of complete microscopic excision, tumor was noted to involve the periosteum of the nasal bones and the lamina papyracia bilaterally. An interdisciplinary approach combining the skills of the dermatologic surgeon and the head and neck surgeon provided the best opportunity for complete eradication of disease by removing the nasal bones and the lamina papyracia bilaterally along with the upper portion of the nasal septum. The extensiveness of the tumor and the history of recurrent disease called for delay of the reconstruction for a period of 18 months to provide ease in observing the region for possible persistent tumor. Indeed, the patient developed a recurrence in the skin adjacent to the nasal defect 1 year after our attempt to cure him of his tumor.

Tumors occurring in the midfacial skin may track along the infraorbital nerve to involve the maxillary sinus or orbit and may extend as far posteriorly as the foramen rotundum. An example of this is seen in Figure 9, which demonstrates a surgical specimen consisting of the maxilla and contents of the orbit. Orbital exenteration was necessary because a recurrent $B C C$ of the facial skin had extended along the infraorbital nerve into the maxillary antrum and orbit regions. Tumor invaded the orbital fat and ex-

\footnotetext{
J. Dermatol. Surg. Oncol. 13:10 October 1987
}

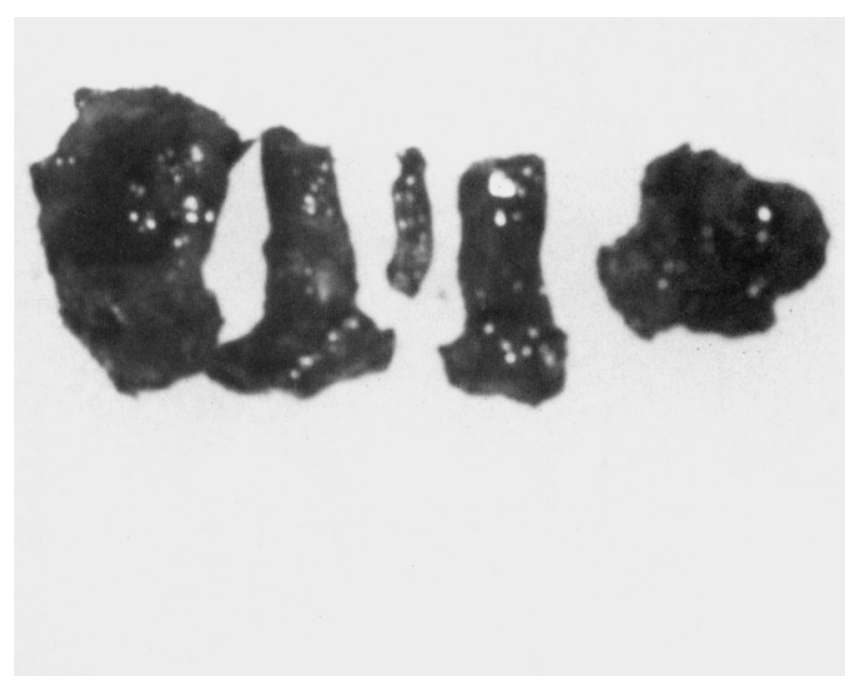

FIGURE 8B. Complete microscopic excision required bilateral ethmoidectomy and resection of the nasal bones and lamina papyracia.

traocular muscles to such a degree that it was not possible to preserve the ophthalmic globe. Tumor also tracked along the infraorbital nerve posteriorly toward the foramen rotundum. Following maxillectomy, frozen section analysis of the nerve exiting the foramen demonstrated malignant cells. Thus it was elected to treat the patient with postoperative radiotherapy. Unfortunately, the patient developed recurrent disease at the skull base and eventually died of his cancer.

This case exemplifies the need for appropriate radiographic studies preoperatively in instances where the clinician is concerned about deep extension of tumor toward the paranasal sinuses. It is also a good example of the aggressiveness manifested by some BCCs occurring in the midfacial region and the need on occasion to use radiation therapy as an adjunct to surgical excision of extensive skin cancers.

\section{SCALP}

BCCs of the scalp tend to occur most commonly in the temple area and may extend deeply to involve the underlying periosteum, temporalis muscle, and calvarium. Extensive tumors may require removal of a large portion of the scalp overlying the frontal and parietal bones. The underlying periosteum is 


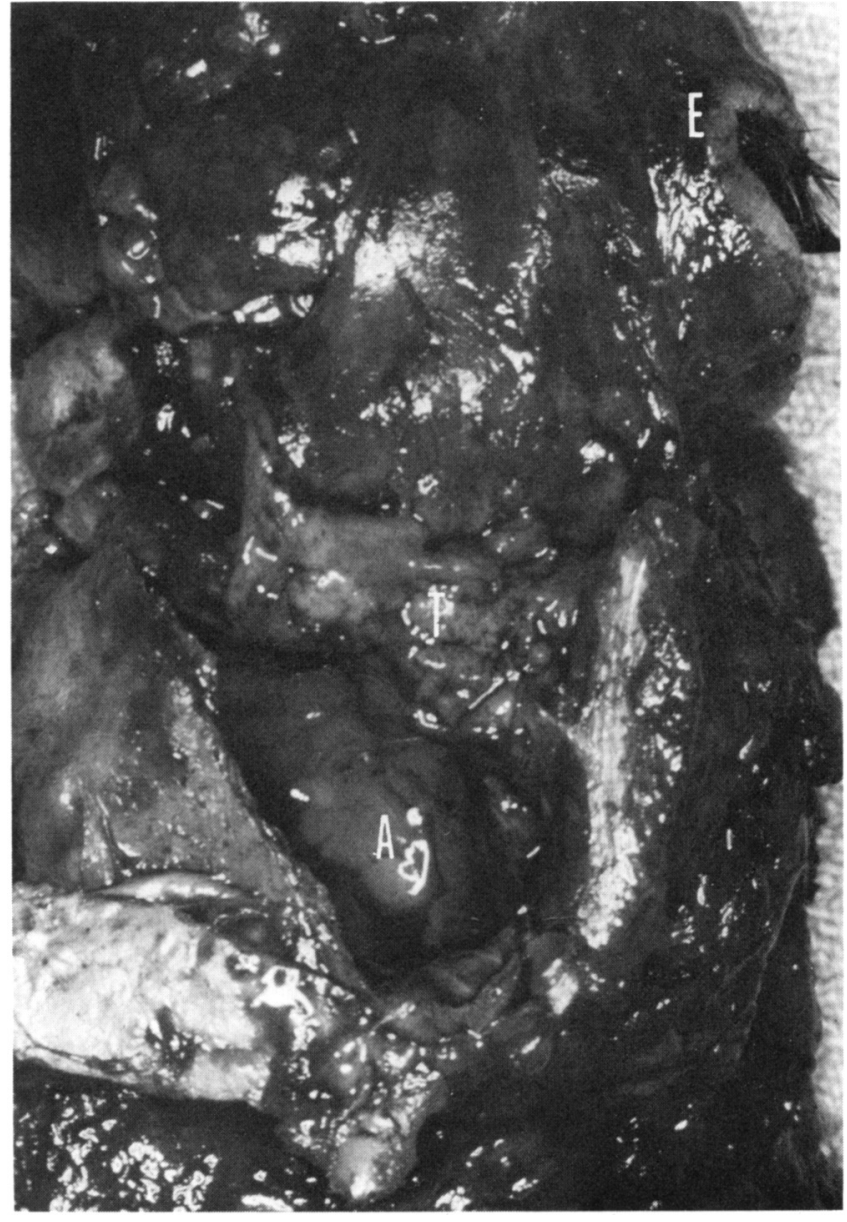

FIGURE 9. Surgical specimen consisting of the maxilla and contents of orbit. BCC involved the entire length of the infraorbital nerve and invaded the orbital fat and extraocular muscles. (E) Eye. (A) Antrum. (T) Tumor involving infraorbital nerve.

frequently involved with neoplasm necessitating removal, thus making reconstruction of such defects more difficult. In instances where periosteum is involved with tumor but no gross tumor is noted to involve the calvarium, the bone is treated by surgical removal of the exterior portion utilizing a rotating cutting burr. It is difficult for a skin graft to heal on bare bone and it may take many months for the defect to granulate and heal by second intention. This is true in spite of drilling holes through the outer cortical bone to facilitate the growth of granulation tissue from the more vascular medullary portion of the calvarium.

Large areas of exposed bone are best treated by rotating a local scalp flap to cover the exposed bone. ${ }^{11}$ The scalp flap is elevated in a plane superficial to the periosteum so that the secondary defect can be closed with a split-thickness skin graft if necessary, or on occasion, may be closed primarily by 1104 local advancement of surrounding scalp tissue. Primary closure is aided by performing incisions through the galea parallel to the major vascular supply to the scalp to enhance stretching of the flap.

Large neglected ulcerative BCCs of the scalp not infrequently invade the underlying calvarium. Tumors that grossly invade the outer cortex of the calvarium require wide full-thickness resection of the cranium. When bone involvement is suspected by the clinician, the patient should be evaluated preoperatively with appropriate radiographic studies including polytomography or CAT scan analysis to assess the cranial bone and underlying brain. Extensive involvement with BCC of cranial bone necessitates well-conceived preoperative planning by the surgical disciplines involved with treating such patients.

Frequently, the dermatologic surgeon will seek the assistance of the head and neck surgeon and the neurosurgeon in resecting such tumors. The multidisciplinary team must be prepared to trace the tumor as deep as necessary in order to eradicate all neoplasm. This may require multiple frozen sections to assess the parameter of the scalp defect, as well as the surgical margins of the dura mater and occasionally the brain itself. The dermatologic surgeon has the capability of processing bone within 24 hours so that bony margins can be readily assessed for the possibility of tumor involvement. When a bony margin is found to contain tumor, the patient is returned to the operating room the next day for additional bone removal.

An example of the interdisciplinary approach for the treatment of large tumors of the scalp is represented in Figure 10. The skills of the dermatologic surgeon and the head and neck surgeon were combined with those of a neurosurgeon to completely eradicate a neglected BCC involving the postauricular scalp. Tumor extended into the mastoid cortex necessitating a modified radical mastoidectomy, removing all of the cortex of the mastoid bone. Fortunately, tumor did not extend into the air cells of the mastoid and thus the facial nerve and hearing were spared. Tumor also extended full thickness through the posterior calvarium and into the underlying dura mater in the vicinity of the lateral sinus.

Tumor invasion of the dura mater over the sinus necessitated removal of the sinus, as well as a large area of dura mater. Complete microscopic survey of the deep surface of the resected dura mater demonstrated no evidence of penetration of tumor into the underlying brain. The defect in the dura mater was repaired with a fascia lata graft and the dural 


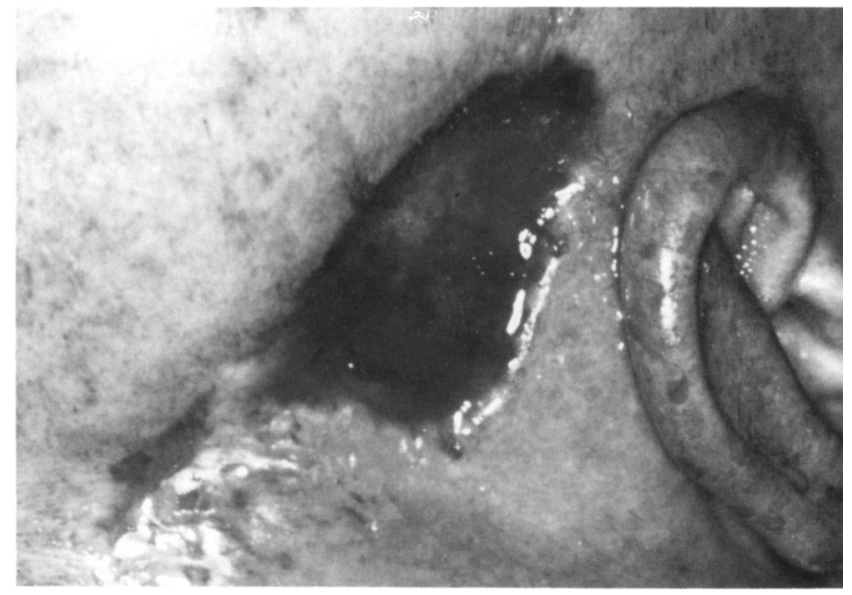

FIGURE 10A. BCC of occipital scalp.

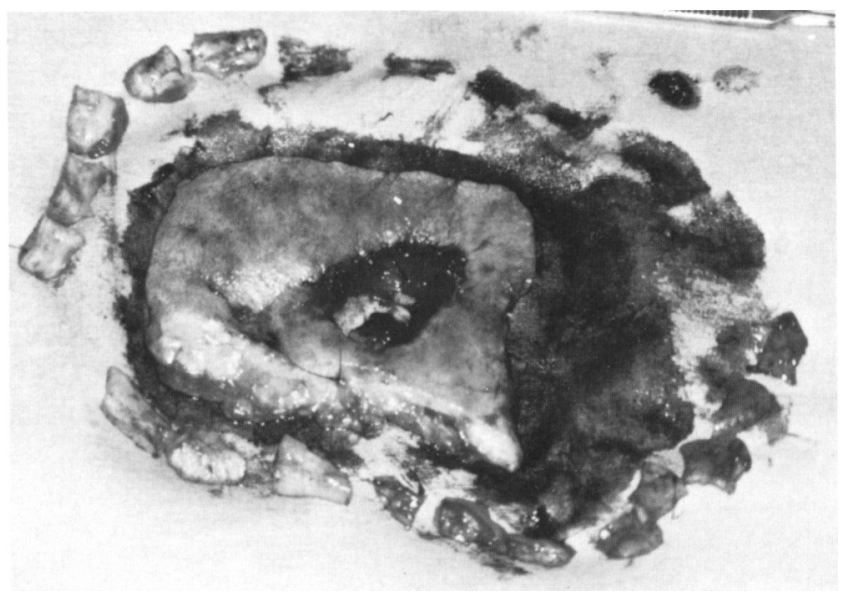

FIGURE 10B. Scalp specimen demonstrating multiple sections necessary to provide complete microscopic control of the surgical margin of the scalp.

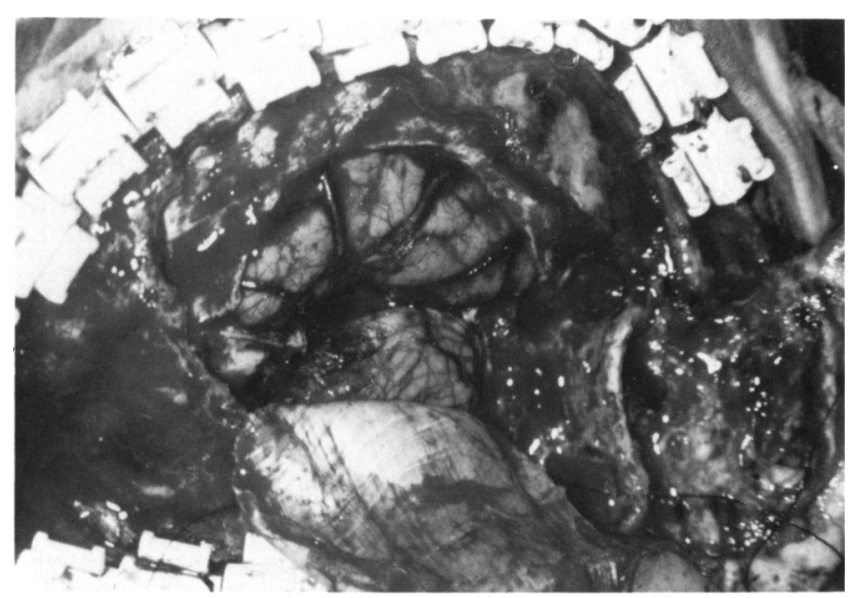

FIGURE 10C. Tumor invasion of the dura mater over the lateral sinus necessitated removal of the sinus as well as a large area of the dura mater. Fascial graft for repair of the dura is attached inferiorly.

J. Dermatol. Surg. Oncol. 13:10 October 1987

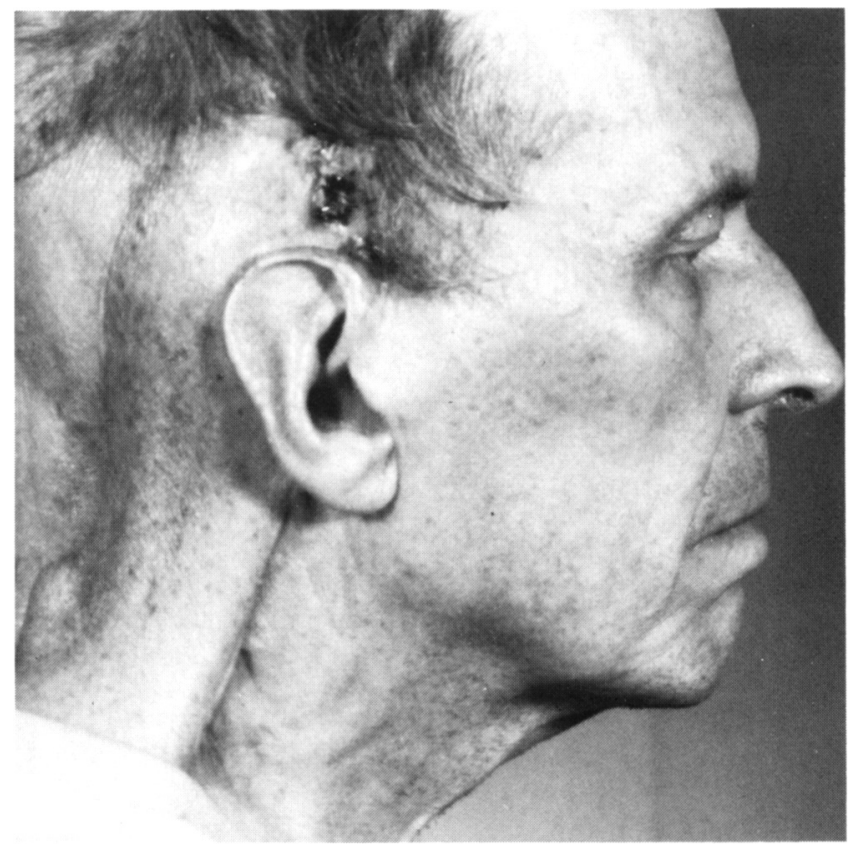

FIGURE 10D. Calverium and fascial graft were covered by a medially based shoulder flap. Six weeks postoperative.

graft and surrounding exposed calvarium were covered with a medially based shoulder flap.

This case highlights the importance of combining the skills of various surgical disciplines to maximize the opportunity of eradicating extensive facial skin malignancies that involve the head and neck region while at the same time providing for the reconstruction of the resulting defect, thus limiting functional impairment.

\section{CONCLUSION}

The usefulness of an interdisciplinary approach combining the skills of the dermatologic surgeon and the head and neck surgeon for the management of extensive BCCs of the head and neck is well founded in our experience. The Mohs surgical technique offers meticulous marginal control for a variety of cutaneous, as well as paracutaneous neoplasms, with the advantage of conserving normal surrounding tissues and structures. Tumor extending deeply from the skin into underlying bone and soft tissue is resected with the assistance of a head and neck surgeon familiar with the anatomy and trained in the protection of the vital structures of the head and neck. The skills of the neurosurgeon are necessary when cancer invades or encroaches upon the central nervous system. It is evident that 
patients with large or aggressive BCCs will best be served when this interdisciplinary approach has become commonplace.

\section{REFERENCES}

1. Levin $\mathrm{H}$, Bailin $\mathrm{P}$, Wood $B$, Tucker $\mathrm{H}$. Tissue conservation in treatment of cutaneous neoplasms of the head and neck: Combined use of Moh's chemosurgical and conventional surgical techniques. Arch Otolaryngol 105:140-144, 1979.

2. Baker SR, Swanson NA. Complete microscopic controlled surgery for head and neck cancer. Head Neck Surg 6:914-920, 1984.

3. Swanson NA, Grekin RC, Baker SR. Mohs surgery: Techniques, indications and applications in head and neck surgery. Head Neck Surg 6:683-692, 1983.

4. Levin HL, Bailin PL. Basal cell carcinoma of the head and neck: Identification of the high risk patient. Laryngoscope XC:955-961, 1980.

5. Bailin PL, Levin HL, Wood BG, Tucker HM. Cutaneous carcinoma of the auricular and preauricular region. Arch Otolaryngol 106:692-696, 1980.

6. Robins P. Chemosurgery: My 15 years of experience. J Dermatol Surg Oncol 7:779-789, 1981.

7. Ceilley RI, Bumstead RM, Smith WH. Malignancies on the external ear: Methods of ablation and reconstruction of defects. J Dermatol Surg Oncol 5:762-767, 1979.

8. Salasche SJ, Amonette R. Morpheaform basal cell epitheliomas: A study of subclinical extensions in a series of 51 cases. J Dermatol Surg Oncol 7:387-392, 1981.

9. Mora CG, Robins P. Basal cell carcinomas in the center of the face: Specific diagnostic, prognostic and therapeutic considerations. J Dermatol Surg Oncol 4:315-321, 1978.

10. Baker SR, Swanson NA. Regional and distant skin flaps in nasal reconstruction. Fac Plast Surg 2:33-44, 1981.

11. Lang NP, Kendrick JH, Flanigin $H$, Wetmore SJ, Suen JY, Westbrook KC. Surgical management of advanced scalp cancer. Head Neck Surg 5:299-305, 1983.

Treatment of Chromomycosis with Liquid Nitrogen. JA de Souza Sittart, NY Valente. Med Cutan Iber Lat Am 14:227-232, 1986 (Portuguese).

Five patients with chromomycosis of the legs from the São Paulo area, Brazil, after unsuccessful attempts of treatment with amphotericin B, 5-fluorocitosine, and ketoconazole, were successfully treated by several applications of liquid nitrogen, with healing accompanied by cicatricization. The evolution of the disease had been from 1 to 25 years. Four patients had verrucous patches and one patient a sarcoid-like lesion. All patients were culture positive for Fonseca pedrosi. There has been no recurrence during a 2 to 4 year follow-up.

YEHUDI M. FELMAN, M.D.
Retinoids and Systemic Chemotherapy in Cases of Advanced Mycosis Fungoides. A Report from the Scandinavian Mycosis Fungoides Group. L Molin, K. Thomsen, G Volden, et al. Acta Derm Venereol 67:179-183, 1987.

Effective therapy for advanced stage mycosis fungoides (MF) is lacking. The authors report their results in 8 patients treated with a combination of bleomycin, cyclophosphamide, and prednisolone and in 12 patients who were treated with these three drugs plus either etretinate (11 patients) or 13-cis-retinoic acid ( 1 patient). All 20 patients had tumor stage MF, and 14 patients had extracutaneous disease. Of the 8 patients receiving combination chemotherapy, 5 showed a partial or complete remission, with a median remission of 3 months. In the patients treated with retinoids, 7 showed a partial or complete response lasting a median of 6 months. The side effects observed were those anticipated and these were usually controlled by a reduction in the dosage of the offending drug. Although this study suggests that retinoids might be beneficial in the management of MF, more patients need to be studied and long-term follow-up is essential (only 1 patient was treated for a year or longer).

PEARON G. LANG, JR., M.D.

Rhodamine-123 as a New Photochemosensitizing Agent with Argon Laser. "Nonthermal" and Thermal Effects on Human Squamous Carcinoma Cells in Vitro. DJ Castro, RE Saxton, HR Fetterman, et al. Laryngoscope 97:554-561, 1987.

Photodynamic therapy (PDT) to manage cancer has recently received much attention. One major drawback, however, is that the photosensitizing agents used make the patient extremely photosensitive. In order to circumvent this problem, the authors studied the effect of the argon laser on cultured human squamous cell carcinoma cells (SCC) which had been incubated with non-toxic doses of Rhodamine-123 (Rh-123). Rh-123 is a mitrochondrial specific dye which is non-photosensitizing and has a peak absorption at $511 \mathrm{~nm}$. The authors found that when SCC cells were incubated with Rh-123 there was an enhancement of the tumoricidal effect of the argon laser. How useful this methodology will be when applied to the clinical situation, however, remains to be demonstrated.

PEARON G. LANG, JR., M.D. 


\section{Erycette (erythromycin 2\%) TOPICAL SOLUTION}

\section{Description:}

Erythromycin is an antibiotic produced from a strain of Streptomyces erythraeus. It is basic and readily forms salts with acids. Each ml of ERYCETTE (erythromycin $2 \%$ ) Topical Solution contains $20 \mathrm{mg}$ of erythromycin base in a vehicle consisting of alcohol (66\%) and propylene glycol. It may contain citric acid to adjust $\mathrm{pH}$.

\section{Actions:}

Although the mechanism by which ERYCETTE

Solution acts in reducing inflammatory lesions of acne vulgaris is unknown, it is presumably due to its antibiotic action

\section{Indications:}

ERYCETTE Solution is indicated for the topical control of acne vulgaris.

\section{Contraindications:}

ERYCETTE Solution is contraindicated in persons who have shown hypersensitivity to any of its ingredients.

\section{Precautions:}

General: The use of antibiotic agents may be

associated with the overgrowth of antibiotic-resistant organisms. If this occurs, administration of this drug should be discontinued and appropriate measures laken.

Information for Patients: ERYCETTE Solution is for external use only and should be kept away from the eyes, nose, mouth, and other mucous membranes. Concomitant topical acne therapy should be used with caution because a cumulative irritant used with caution because a cumulative irritant desquamating, or abrasive agents.

Carcinogenesis, Mutagenesis, Impairment of

Fertility: Long-term animal studies to evaluate carcinogenic potential, mutagenicity, or the effect on fertility of erythromycin have not been performed. Pregnancy: Pregnancy Category C. Animal reproduction studies have not been conducted with erythromycin. It is also not known whether erythromycin can cause fetal harm wher ering regnant woman or can affect eproduction capacity. Erythromycin should be given to a pregnant woman only if clearly needed Nursing Mothers: It is not known whether erythromycin is excreted in human milk after topical application. However, this is reported to occur with oral and parenteral administration. Therefore caution should be exercised when erythromycin is administered to a nursing woman.

\section{Adverse Reactions:}

Adverse conditions reported include dryness tenderness, pruritus, desquamation, erythema. oiliness, and burning sensation. Irritation of the eyes has also been reported. A case of generalized urticarial reaction, possibly related to the drug, which required the use of systemic steroid therapy has been reported.

\section{Dosace and Administration:}

The ERYCETTE pledget should be rubbed over the affected area twice a day after the skin is thoroughly washed with warm water and soap and patted dry.

Acne lesions on the face, neck, shoulder, chest, and back may be treated in this manner. Additional pledgets may be used, if needed.

\section{How Supplied:}

ERYCETTE (erythromycin 2\%) Topical Solution is supplied as foil-covered saturated pledgets (swabs) in boxes of 60 (NDC 0062-1185-01)

Store at controlled room temperature $\left(59^{\circ}-86^{\circ} \mathrm{F}\right)$.

Manufactured for

DERMATOLOGICAL DIVISION ORTHO PHARMACEUTICAL CORPORATION

Raritan, New Jersey 08869-0602

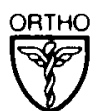

Subungual Melanoma: A 25-Year Review of Cases. JM Daly, R Berlin, C Urmacher. J Surg Oncol 35:107-112, 1987.

A retrospective study looking at the clinical pathologic stage of disease in 33 patients with subungual melanoma was evaluated from patients seen at Memorial Sloan-Kettering Cancer Center between the years 1950 to 1975 . Females (22) outnumbered males (11) and the tumors were equally distributed between the hand and foot. The most common anatomic locations were the thumb and large toe. Of 16 patients with hand subungual disease, 11 were stage I and 3 were stage II. None of the stage I individuals undergoing elective node dissection had microscopic evidence of disease where as all of the stage II patients had lymph node involvement. Seventeen patients had toes subungual melanomas, 10 were clinically stage I and 7 were clinically stage II. Of 9 stage I patients undergoing lymph node dissection, 3 had microscopic lymph node disease. The 5- and 10-year survivals were 66 and $55 \%$ for clinical stage I disease versus 22 and $0 \%$ for clinical stage II disease. Pathologic stage I disease showed 5- and 10-year survivals of 79 and $61 \%$ versus 18 and $9 \%$ for stage II disease. Site did not influence survival when controlled for pathologic stage. Histologic grading was attempted, but no firm conclusions could be drawn except that the thicker tumors appear to have a worse prognosis. Prognosis was closely related to the clinical/pathologic stage of the disease.

R. STEVEN PADILLA, M.D.

Excision of Large Basal Cell Carcinomas - Problems and Planning. $H$ Breuninger. $Z$. Hautkr 62:269-279, 1987 (German).

During the excision of 1083 basal cell carcinomas, the clinically detectable diameters and the safe margins along the tumor border were calculated. The excision edges were histologically controlled. 115 of the 1083 tumors showed diameters exceeding $20 \mathrm{~mm}$. The frequency of excisions with negative or positive edges corresponded to the safe margin and depth of the incision. The results showed that even for large tumors a safe margin of more than $5 \mathrm{~mm}$ is seldom appropriate or necessary. The excision should always include the subcutaneous tissue as well as the underlying tissue, if the stite of the lesion permits it. The excised edges should be histologically controlled if radical excision is contemplated. 